\title{
In-Vivo Pan/Tilt Endoscope with Integrated Light Source
}

\author{
Tie Hu, Peter K. Allen and Dennis L. Fowler
}

\begin{abstract}
Endoscopic imaging is still dominated by the paradigm of pushing long sticks into small openings. This approach has a number of limitations for minimal access surgery, such as narrow angle imaging, limited workspace, counterintuitive motions and additional incisions for the endoscpic instruments. Our intent is to go beyond this paradigm, and remotize sensors and effectors directly into the body cavity. To this end, we have developed a prototype of a novel insertable pan/tilt endoscopic camera with an integrated light source. The package has a size of $110 \mathrm{~mm}$ in length and $10 \mathrm{~mm}$ in diameter and can be inserted into the abdomen through a standard trocar and then anchored onto the abdominal wall, leaving the incision port open for access. The camera package contains three parts: an imaging module, an illumination module, and a pan/tilt motion platform. The imaging module includes a lens and CCD imaging sensor. The illumination module attaches to the imaging module and has an array of LED light sources. The pan/tilt platform provides the imaging module with pan of 120 degrees and tilt motion of 90 degrees using small servo motors. A fixing mechanism is designed to hold the device in the cavity. A standard joy stick can be used to control the motion of the camera in a natural way. The design allows for multiple camera packages to be inserted through a single incision as well.
\end{abstract}

\section{INTRODUCTION}

Our goal is to enhance and improve surgical procedures by placing small, mobile, multi-function platforms inside the body that can begin to assume some of the tasks associated with surgery. We want to create a feedback loop between new, insertable sensor technology and effectors we are developing, with both surgeons and computers in the informationprocessing/control loop. We envision surgery in the future as radically different from today. This is clearly a trend that has been well-established as minimal-access surgical procedures continue to expand. Accompanying this expansion has been new thrusts in computer and robotic technologies that make automated surgery, if not feasible, an approachable goal. It is not difficult to foresee teams of insertable robots performing surgical tasks inside the body under both surgeon and computer control. The benefits of such an approach are well documented: greater precision, less trauma to the patient, and improved outcomes. One factor limiting this expansion is that the laparoscopic paradigm of pushing long sticks into small openings is still the state-of-the-art. While this paradigm has been enormously successful, and has spurred development of new methods and devices, it is ultimately limiting in what it

This work was supported by NIH grant 1R21EB004999-01A1.

Tie Hu (Ph.D) is with Department of Computer Science, Columbia University, New York, NY10027 USA tiedcs . columbia. edu

Peter K. Allen (Ph.D) is with Department of Computer Science, Columbia University New York, NY 10027 USA allen@CS . columbia.edu

Dennis L. Fowler (M.D) is with Department of Surgery, Columbia University New York, NY 10032 USA dlf91@columbia.edu can achieve. Our intent is to go beyond this paradigm, and remotize sensors and effectors into the body cavity where they can perform surgical and imaging tasks unfettered by traditional endoscopic instrument design.

In laparoscopic surgery, the surgeon first cuts several small incisions in the abdomen, and inserts trocars (small tubes) through the holes. Then they insert the laparoscopic instruments and endoscopic camera into the abdomen through the trocars. The abdomen is inflated by carbon dioxide gas in order to provide the space for the operation and vision. By viewing the image from the endoscopic camera, the surgeons operate the laparoscopic tools to perform surgery. Laparoscopic surgery has several benefits, such as smaller and fewer incisions, less pain and trauma to the patients, faster recovery time, and lower health care cost. However, this new technique drastically increases the complexity of a surgeons' operation because of the rigid, sticklike instruments, impaired depth perception, loss of sense of touch and the difficulty in varying the perspective view of the operative field [1]. As a critical imaging device, the basic architecture of the endoscope has not been fundamentally changed since the invention of the rod-lens by Hopkins and cold light source of fiber optics by Karl Storz in 1950's[2]. Traditional endoscope uses the fiber-optics to deliver the light into the abdomen and the rodlens to transmit the image back to the CCD camera sensor. This approach has a number of limitations, such as narrow imaging, limited work space, counter intuitive motion and additional incisions for the endoscope. Since the surgeon is generally working with both hands holding other instruments, an assistant is necessary to hold the endoscope steady and move it as required. Recent work in robotics has sought to automate that task. One commercially available system called AESOP can orient a traditional endoscope using a robotic arm that is controlled by spoken commands[3]. While this takes the burden off the assistant and provides a much more stable image, it still occupies a large part of the operating room floor. The similar principle is used in da Vinci surgical robots[4] . A simpler robotic endoscope manipulator that can be placed directly over the insertion point was developed at INRIA[5]. However, none of these systems addresses the fundamentally limited range of motion of the endoscope. The fulcrum point created by the abdominal wall restricts the motion of the scope to 4 degrees of freedom, so that the only translation possible is along the camera axis.

There is some related research on new designs for endoscopes. One system uses a traditional rigid rod endoscope but adds a motor that rotates a 90-degree mirror at the end of the scope to provide an additional degree of freedom [6]. Another system is essentially a multi-link arm that positions 


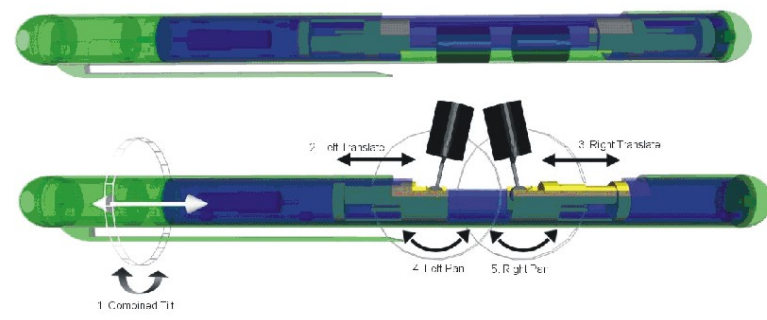

Fig. 1. Design of 5-DOF insertable camera device

a camera using piezoelectric actuators [7]. Theoretically this robot would provide many different viewing angles for an attached camera, but the authors provide no information about the safety of using piezoelectoric electric elements, and do not appear to have attempted any tests within living animals or humans. The pill camera [8] is an example of a camera that operates entirely within the body. It is able to image sections of the small intestine that an endoscoope cannot reach. However, it does not have any means of actuation and simply relies on peristalsis for locomotion. Magnetic anchoring was used to maneuver the locomotion of a micro camera in the body [9]. Since there are no additional actuators in the camera, the view point is limited by the camera orientation.

\section{PROTOTYPE I}

In earlier work [10] we designed a robotically actuated, multi-camera 5 degree of freedom (DOF) system that can be inserted entirely within the abdominal cavity. Once inserted, the device is rigidly fixed to the interior abdominal wall to provide a stable base for the actuated cameras. After situating the device near the operation site, the cameras can be extracted, and look upon the area of interest.

Figure 1 contains a) an image of the simulated device with cameras retracted for body cavity insertion, b) the device with cameras opened for imaging. The design of the device allows it to be fully inserted into the body cavity through a traditional laparoscopic incision. A video of device is available at:http://www1.cs.columbia.edu/ allen/invivosages.mpg.

To test this concept, we have built a single camera prototype with 3 DOF (pan, tilt, translation). Figure 2 shows the different degrees-of-freedom. Figure 3 shows the initial tracking system we have developed to have the camera follow a target automatically. This tracker uses image-based error to reposition the camera and keep the target centered in the field-of-view. Figure 4 shows an initial experiment in a laparoscopic training box. The device was mounted inside the box, and a surgeon was able to perform a simulated suturing task using the image from the prototype device. The camera was manually controlled by an assistant using a joystick responding to the surgeon's instructions. Figure 5 shows the device being moved to explore the entire viewing field, allowing the surgeon to see over a greater distance than traditional laparoscopes.

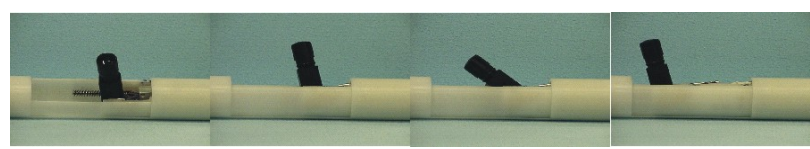

Fig. 2. Initial prototype of in-vivo imaging device. This prototype has a single camera, but the platform is designed to have 2 cameras. This sequence demonstrates the motions that can be achieved for a single camera. First tilting about the central device axis is shown, then panning about an orthogonal axis, and finally translation. The second camera will have a common tilt axis and independent pan and translation axes.

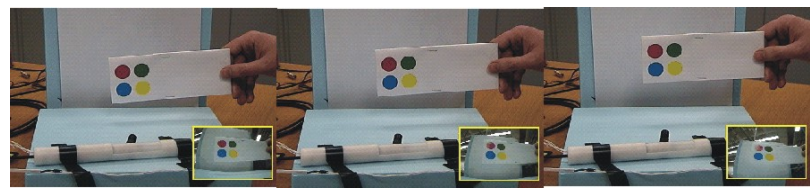

Fig. 3. Early visual servoing experiments demonstrate that the system can keep a moving target pattern within its field of view. Image-based visual servoing is used to track the target automatically.

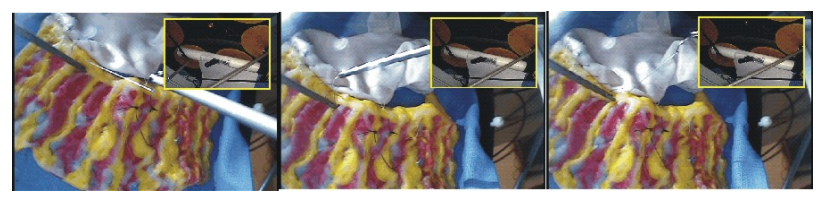

Fig. 4. The device was used in a suturing experiment within a laparoscopic training box. The image provided by the device was sufficiently clear to perform the task without using any additional image sources. The surgeon's assistant was able to adjust the view at the surgeon's request by joystick control of the camera's pan, tilt and translation axes.

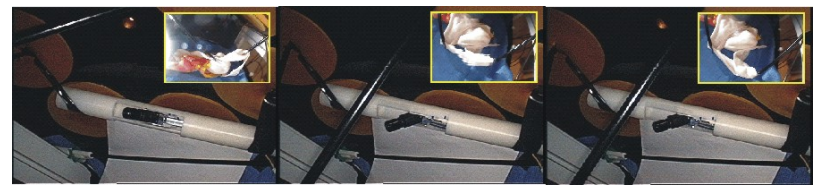

Fig. 5. Another task, that required motions over greater distances, was performed within the training box. Again the assistant was able to keep the activity within the field of view of the camera. This sequence more clearly shows the motions of the camera.

The outer shell of the prototype device is a tube that is $22 \mathrm{~mm}$ in diameter, $19 \mathrm{~cm}$ long (see figure 2), and the cabling emerges from the proximal end. The first motor, which controls the tilting motion of the cameras, is parallel with the central axis of the shell and is near the proximal end. This motor rotates an inner shell that contains both cameras and the other motors. A $5.8 \mathrm{~cm}$ long section of the outer tube is cut away at the distal end to allow the cameras to tilt 180 degrees when they are extracted.

This device was tested in a mock up using a surgical training box. In this study, we compared the performance of six surgeons in the surgical mock up [11]. The surgeons each completed twice a series of five validated tests (MISTELS: McGill Inanimate System for the Training and Evaluation of Laparoscopic Skill). Each surgeon completed the series of tests using the prototype imaging platform once and a standard video laparoscopy system once. The performance of each surgeon using the new imaging platform was compared to his/her performance using the laparoscope. There was no 


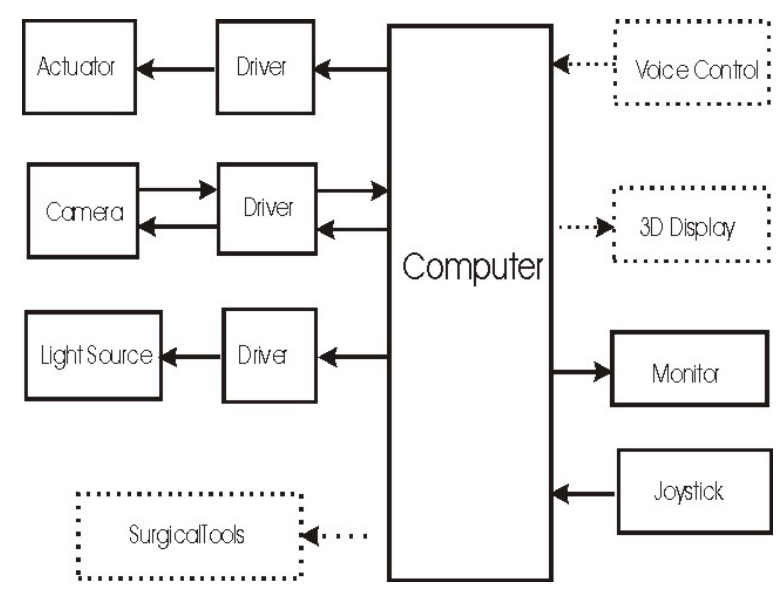

Fig. 6. System Configuration of Motorized Endoscope

significant difference in the performance on the tests for any surgeon using the two imaging systems.

\section{PROTOTYPE II}

Our work with Prototype I has led us to design a second generation device that improves upon the design of the initial device. Our design goals for Prototype II included reducing the device size and the inclusion of an integrated light source. To reduce the device size to allow it to be inserted through a $12 \mathrm{~mm}$ trocar, we removed 1 camera and the translation axis. We have also added an LED light source to the device.

We make use of modular design to make the device components interchangeable and extendable. The current system includes a user-friendly interface, making it easier to control the camera's DOF using natural motions. It consists of a Pan/Tilt motorized CCD camera with illumination components, control interface driver, PC, and Joystick controller. An anchoring mechanism has been designed to attach the device to the abdominal wall. After the surgeon anchors the camera onto the abdomen wall, he can use the Joystick to position the camera to the desired surgical viewpoint using the Pan and Tilt motions. The intensity of illumination can be adjusted manually through the control panel. Figure 6 shows the configuration of the system. The solid-line blocks show the current system's functions. The dot-line blocks show the extendable functions in the future. The computer is a standard PC with NTSC frame grabber for video and a D/A card for implementing control voltages to the pan tilt motors. Figure 7 shows a CAD model of the device. Figure 8 shows images of the implemented prototype device, with integrated lighting and pan/tilt axes. Advanced camera control and tracking algorithms can also be implemented in the system.

\section{A. Design of Light Source}

Most endoscopes use a Xenon light source and fiber optics to illuminate the internal body. This method consumes power and is costly. In addition. fiber optics are not suitable for our device because of their fragility when subjected to large bending/rotation moments. LED's have been used

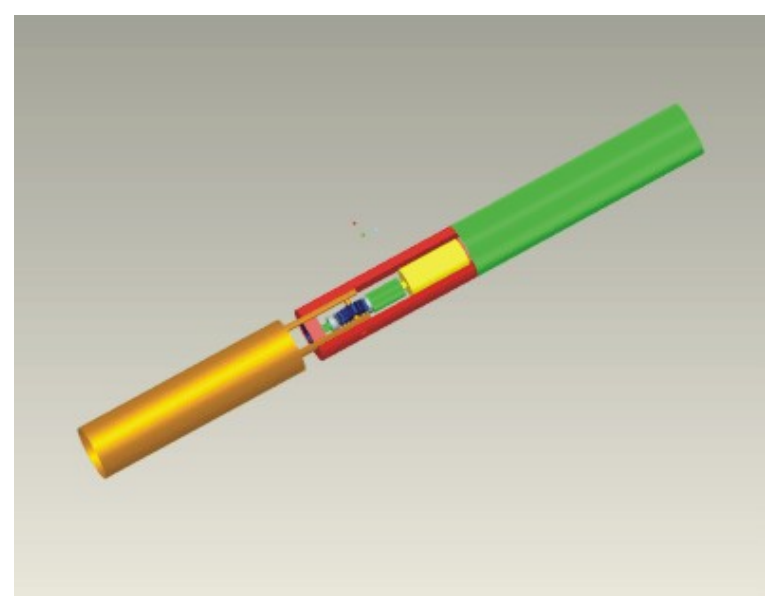

Fig. 7. CAD model of device

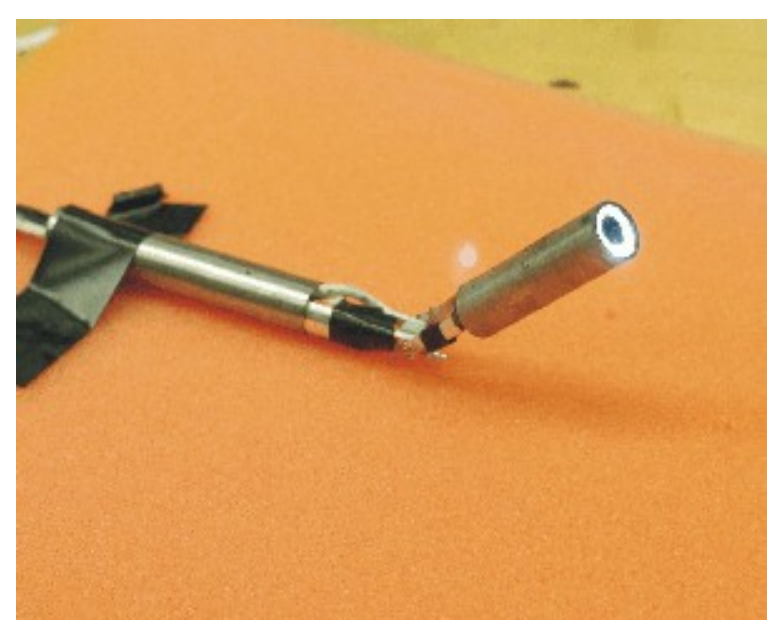

Fig. 8. Implemented Prototype II device with LED lighting and pan/tilt axes.

as light sources for medical devices in the past, and they have the advantage of lower power, higher efficiency, lower cost, smaller package size and longer lifespan. We selected Luxeon Portable PWT white LED (LXCL_PWT1) as the illumination unit of the device. It has a small package size of 2.0X1.6X0.7 mm, which can generate 26 lumens of light at $350 \mathrm{~mA}$, with a color temperature of $6500 \mathrm{~K}$. The lifespan is about 2,000 hours. The illumination unit is a custom made PCB(printed circuit board) board with 8 LEDs. It has a size of $9 \mathrm{~mm}$ in external diameter, $5 \mathrm{~mm}$ in internal diameter, and $3 \mathrm{~mm}$ in thickness. The 8 LED's are serially connected and soldered in a circular printed circuit board. It can deliver a total of 208 lumens of light, with a power consumption of $8.4 \mathrm{~W}$, which is less than Xenon light source's $170 \mathrm{~W}$ power consumption. Figure 9-Up) shows the CAD layout of LED ring. A PCB plate was printed with an array of LED ring. A circular plate with one ring was cut and machined into a $9 \mathrm{~mm}$ circular plate. Then it was drilled with a $5 \mathrm{~mm}$ hole in the center. Figure 9-Down) shows the finished LED board which can be soldered with the LED's. The internal hole of the board works also as an aperture. The LED ring is inserted 


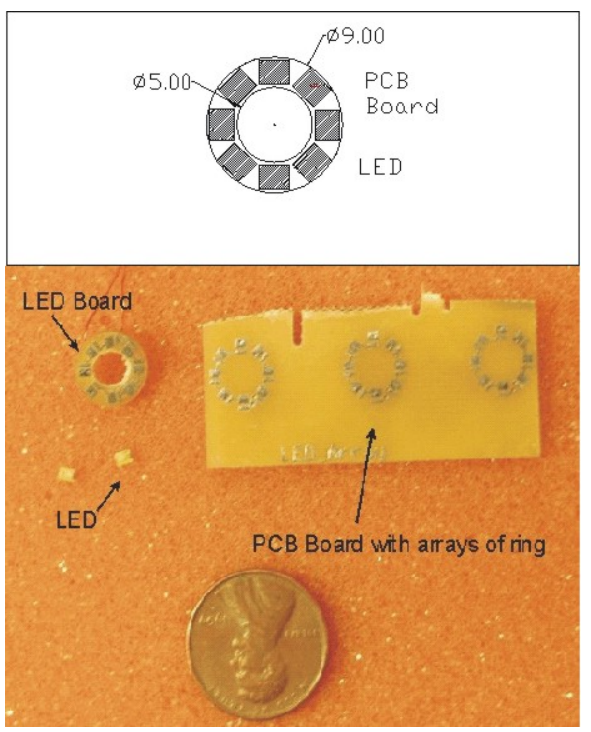

Fig. 9. Up: CAD Layout of LED Down: LED board and LED

into a camera module with $10 \mathrm{~mm}$ diameter and fixed in front of lens. An aluminum tube is used as the external shell of the camera module to quickly dissipate the heat generated by the LEDs.

\section{B. Lens and Camera Design}

A standard endoscope uses a series of relay lenses to transmit the image to the CCD camera sensor outside of the body. This approach protects the fragile electronics from the body fluid and moisture. However, the complicated optics and mechanical structure increases the cost of a standard endoscope. It makes it almost impossible to be a disposable device. The advantage of our approach is that the standard CCD camera and lens can be used in our device, which can reduce the direct cost of the device, making the unit lowcost and potentially disposable. One scenario is to dispose of low-cost components, such as the lens and mechanical components while saving the expensive parts such as CCD camera head and motors after operations. In our device, sealing has two functions, one for the protection, and the other to recycle the expensive components for future use.

A custom-made lens is a better choice to meet the specific optics design needed. If we consider the view distance (the distance between the lens and image object) as $150 \mathrm{~mm}$ to $200 \mathrm{~mm}$, and the view angle as 50 degree, we can calculate the focal length and diameter of the lens. The standard CCD sensor's active view is $3.6 \times 2.7 \mathrm{~mm}$, which equals to the active view of circle with diameter in $4.5 \mathrm{~mm}$. F number is chosen as 4.

F number $\mathrm{N}$ is defined as: $N=\frac{F}{D}$ [12]

Here, $f$ is focal length and $\mathrm{D}$ is the diameter of lens.

$$
f=\frac{2.25}{\tan (25 \mathrm{deg})}=4.825 \mathrm{~mm}
$$

Therefore, we get the diameter of the lens

$$
D=\frac{f}{N}=\frac{4.825}{4}=1.206 \mathrm{~mm}
$$

We use a miniature pin-hole lens (PTS 5.0 from Universe Kogaku America) with appropriate optical parameters for our device (Figure 11-Left). The focal length of the lens is 5.0 $\mathrm{mm}$ and $\mathrm{F}$ number is 4.0. The external casing of the lens was machined to $9 \mathrm{~mm}$.

In our initial prototype, our camera head was the STCR640 produced by Sentech. It uses an $8 \mathrm{~mm}$ round CCD package containing a " full resolution (768 x 494) color image sensor. Other miniature CCD or CMOS cameras have been developed for medical application in recent years. Medigus (Medigus Inc) claims the smallest CCD camera in the world with the diameter of $1.8 \mathrm{~mm}$ and integrated optics, which has been implemented in an endoscope package. Panasonic also has a $1.8 \mathrm{~mm}$ CCD camera (GPKS2MM) with resolution of 537(H) X 505(V). We use a " color video CCD camera head (Figure 11-Right) with diameter of $6.5 \mathrm{~mm}$ (NET USA Inc, CSH-1.4-V4-END-R1) in this package. The camera has active pixels of $752(\mathrm{H}) \times 582(\mathrm{~V})$ at PAL system, which can provide $450 \mathrm{TV}$ lines in horizontal resolution and 420 TV lines in vertical resolution. This camera has a standard resolution of " CCD sensor and a smaller package size.

Figure 10 shows the assembly of the camera module. The external shell of camera module is an aluminum tube with external diameter of $10 \mathrm{~mm}$ and wall thickness of 0.25 $\mathrm{mm}$. Aluminum has high thermal conductivity. It can quickly dissipate the heat generate by LED light source. The CCD camera head is packed in a PEEK (a FDA approved plastic) tube with external diameter of $9 \mathrm{~mm}$ and internal diameter of $6.5 \mathrm{~mm}$. Two semicircular parts tightly clamp the end of CCD camera head wire. This design packages the fragile soldering point of the camera and insulates the terminator of head from the aluminum tube. A PEEK tube is fixed between the pin hole lens and the CCD camera head. The length of the tube is exactly same as the focal length of the lens. Therefore, the image can be perfectly projected onto the CCD image sensor. The LED light source is put in front of the lens. Finally, a sapphire (Edmund Optics $9.5 \mathrm{~mm}$ ) is put in front of LED and sealed with epoxy glue.

\section{Pan and Tilt Mechanism design and Packaging}

The pan/tilt actuators are smoovy motors from FaulhaberGroup. The brushless DC motor (0513G) with 625:1 planetary gearhead(Series 06A) has a length in $27 \mathrm{~mm}$ and a diameter of $5.8 \mathrm{~mm}$. It can deliver a torque of 25.0 $\mathrm{mNm}$ at continuous operation and $37.5 \mathrm{mNm}$ at intermittent operation. Two motors are used in one device. One drives the worm gear for Pan motion and the other directly drives the Tilt motion. The worm gear (KLEISS Gear, Inc) has a gear reduction ratio of 16:1. The worm gear mechanism can transverse the motion in a compact size and increase the output torque. Figure 13 shows a wire frame CAD model 


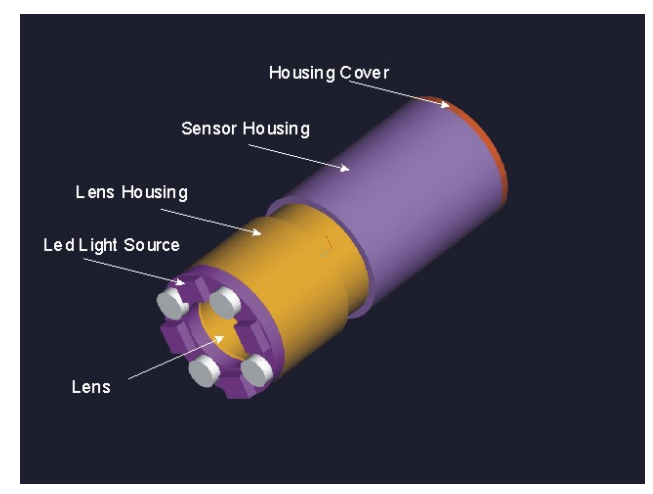

Fig. 10. Assembly of camera module and lens

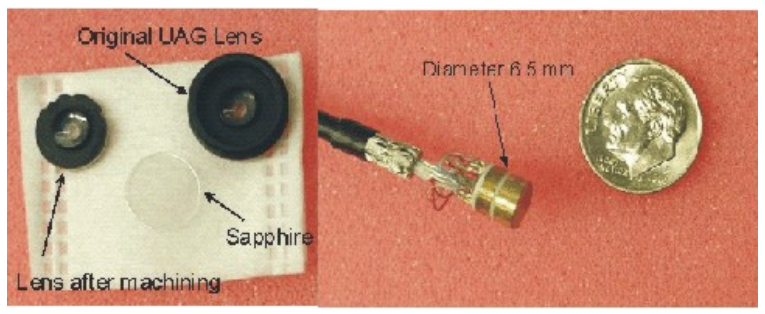

Fig. 11. Left: Lens and Sapphire, Right: CCD Camera Head

of the device. The motors are fixed in the internal part of device by set screws. A coupler connects the Pan motor axis and the worm. The other end of worm is supported by a bearing so that the motor axis could be kept aligned with the worm axis. A gear is transversely rotated through the movement of the worm. The camera module is linked with the axis of the gear by a joint. Therefore, it can rotate as the pan motor moves. The Tilt motor is coaxially aligned with the external shell and directly drives the external shell through a coupler. If the external shell is fixed on the wall of abdomen, the camera module will tilt as tilt motor rotates. The motor wires exit from the side of tilt motor coupler. The terminators of the motors were remade to fit into a $10 \mathrm{~mm}$ package. Figure 12-Left shows the modified motor terminal. Three magnetic wires are soldered into the three terminators of the motor. Then epoxy glue sealed the soldering point.

\section{Fixing the device to the wall of abdomen}

We studied several ways to fix the device onto the wall of the abdomen. The first method is to rely on the suturing

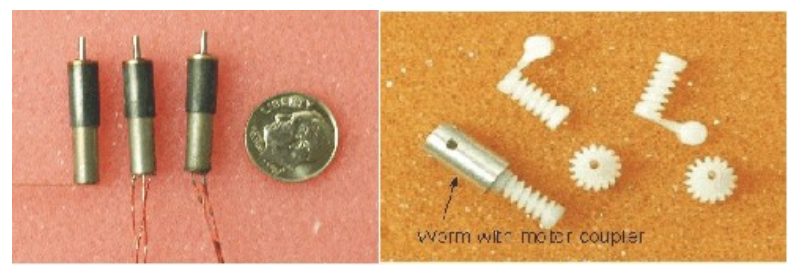

Fig. 12. Left: Smoovy motor with magnetic wire, Right: Worm gear

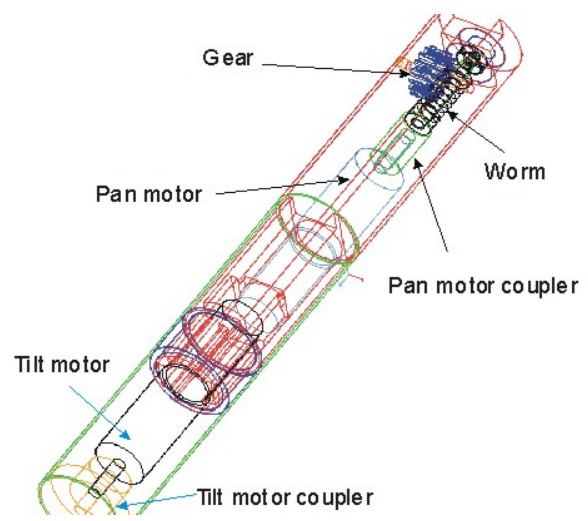

Fig. 13. CAD Model of Pan/Tilt motion platform

skills of surgeons. We made two groves on the surface of the external shell. The width of each grove is about $1 \mathrm{~mm}$ and the depth is about $0.1 \mathrm{~mm}$. When the device is deployed into the abdomen, a surgeon can use braided silk to suture the device onto the abdomen wall. The second way is to design and make a holding mechanism. This mechanism has rotational attachment which holds the tilt motor end of the device. When the surgeon grasps the handle of the mechanism, this attachment can rotate about 90 degrees. After the device is deployed into the abdomen, the surgeon can pull the handle and rotate the device 90 degrees. We have tried both these methods in animal experiments. Another possible method is to use magnetic anchoring. Two internal magnetic pads can be installed in the ends of device. When the device is fully deployed into the abdomen, the surgeon can use external magnetic components to maneuver the locomotion of device outside of body. In this way, we can omit the translation freedom of device so that the size can be minimized. Another advantage is the non-invasiveness of this method. However, the intensity of the magnetic field will decrease with the increase of the abdomen's thickness [9].

\section{RESUlTS}

A porcine animal test was performed to test the Prototype II. The surgeon first cut several small incisions in the abdomen and inserted the trocars. The abdomen of the porcine was inflated with carbon dioxide gas. A standard endoscope was inserted for the purpose of comparison and visually recording the new device inside the abdomen. The surgeon inserted the new device into the abdomen through a trocar. Figure 9 shows the image from the endoscope (left) showing the new device inside the abdomen, along with camera image from the device (right). Further animal tests, which are ongoing, will test the efficacy of this new design.

\section{CONCLUSIONS AND FUTURE WORKS}

This paper describes the design and construction of two prototype in-vivo imaging devices for laparoscopic surgery. 


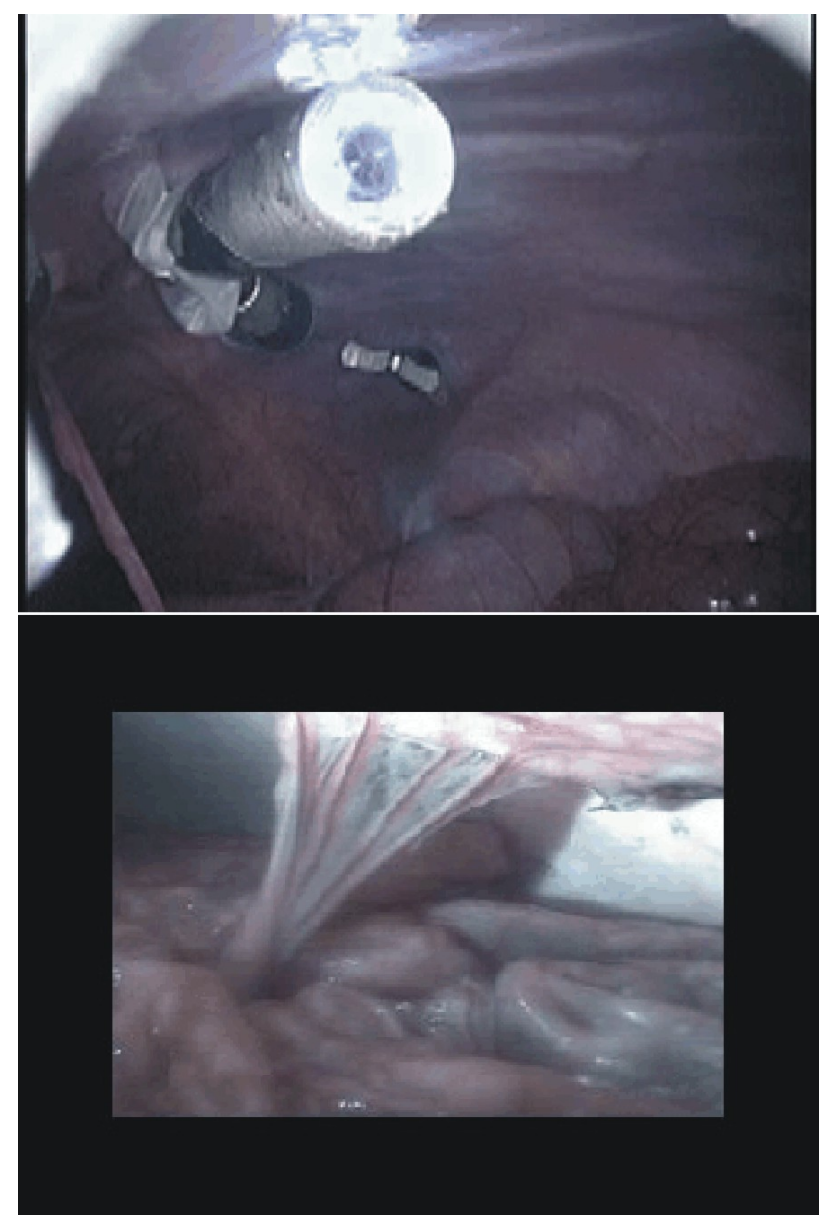

Fig. 14. Up: Image from a standard laparoscope showing Prototype II inside the abdomen. Down: Image from inside the abdomen using Prototype II camera system

The intent is to create totally insertable surgical imaging systems which do not require a dedicated surgical port, and allow more flexibility and DOF's for viewing. Prototype I includes pan, tilt and translation axes, and has been tested in a surgical trainer. Prototype II has a reduced package size and integrated lighting, and is currently being evaluated in in-vivo animal experiments.

We believe these insertable platforms will be an integral part of future surgical systems. The platforms can be used with tooling as well as imaging systems, allowing some surgical procedures to be done using such a platform. The system can be extended to a multi-functional surgical robot with detachable end-effectors (grasper, cutting, dissection and scissor). Because the systems are insertable, a single surgical port can be used to introduce multiple imaging and tooling platforms into a patient.

The current design can be improved in a number of ways. Automatic focusing and zoom capability are desirable for many surgical procedures. The anchoring systems described above all need to be thoroughly tested to allow a stable base for the device.

One of our design goals is to simplify the operation and control of the imaging system. One possible approach to controlling the cameras would be to use a hybrid controller, which allows the surgeon to control some of the degreesof-freedom (DOF) of the device and an autonomous system, which controls the remaining DOF. For example, the autonomous system can control pan/tilt on the camera to keep a surgeon-identified organ in view, while the surgeon simultaneously may translate the camera to obtain a better viewing angle - all the while keeping the organ centered in the viewing field. We have developed hybrid controllers and mechanisms similar to this for robotic work-cell inspection [13] and believe we can transfer these methods for use with this device.

\section{ACKNOWLEDGMENTS}

We thank Nancy Hogle for her help in project development and lab support. This work was supported by NIH grant 1R21EB004999-01A1.

\section{REFERENCES}

[1] R. H. Taylor, etl, ComputerIntegrated Surgery: Technology and Clinical Applications Cambrisge, MA: The MIT Press; 1996.

[2] G. J. Fushs, "Milestones in endoscope design for minimally invasive urologic surgery: the sentinel role of a pioneer," Surg Endosc, vol. 20, pp. 493-499, 2006.

[3] W. P. Geis, H. C. Kim, E. J. B. Jr., P. C. McAfee, and Y. Wang, "Robotic arm enhancement to accommodate improved efficiency and decreased resource utilization in complex minimally invasive surgical procedures," Proc. Medicine Meets Virtual Reality: Health Care in the Information Age,pp.471-481, 1996.

[4] G. Guthart and K. Salisbury, "The IntuitiveTM Telesurgery System: Overview and Application," IEEE International Conference on Robotics and Automation,pp.618-621, 2000.

[5] P. Berkelman, P. Cinquin, J. Troccaz, J. Ayoubi, C. Letoublon, and F. Bouchard, "A compact, compliant laparoscopic endoscope manipulator," IEEE Intl. Conf. on Robotics and Automation,pp.1870-1875, 2002

[6] L. M. Gao, Y. Chen, L. M. Lin, and G. Z. Yan, "Micro motor based a new type of endoscope," Intl. Conf of the IEEE Engineering in Medicine and Biology Society,vol.20(4),pp.1822-1825, 1998.

[7] K. Ikuta, M. Nokata, and S. Aritomi, "Biomedical micro robots driven by miniature cybernetic actuator," IEEE Workshop on Micro Electromechanical Systems, pp.263-268, 1994.

[8] M. Yu, "M2A? Capsule endoscopy: A breakthrough diagnostic tool for small intestine imaging," Gastroenterology Nursing, vol. 25, pp. pp. 24-27, 2002.

[9] S. Park, R. Bergs, R. Eberhart, L. Baker, R. Fernandez, and J. A. Cadeddu, "Trocar-less Instrumentation for Laparoscopy Magnetic Positioning of Intra-abdominal Camera and Retractor," Annals of Surgery, vol. 245,Issue.3,pp.379-384, 2007.

[10] A. Miller, P. Allen, and D. Fowler, "In-Vivo Stereoscopic Imaging System with 5 Degrees-of-Freedom for Minimal Access Surgery," Medicine Meets Virtual Reality Conference (MMVR), Newport Beach, CA, 2004.

[11] V. E. M. Strong, N. J. Hogle, and D. L. Fowler, "Efficacy of Novel Robotic Camera vs a Standard Laparoscopic Camera," Surgical Innovation, vol. 12, 2005.

[12] W. J. Smith, Modern Lens Design, McGraw-Hill, 2004

[13] P.Oh and P.Allen, "Visual Servoing by Partitioning Degrees-ofFreedom," IEEE Trans. on Robotics and Automation, vol. 17, pp. pp.117, February 2001. 